Patricia Hartwich

Anna Małgorzewicz

Universität Wrocław, Wrocław

DOI: $10.19195 / 0435-5865.141 .28$

\title{
Kooperatives Übersetzen bei der Filmübersetzung - translationsdidaktische Überlegungen und Erfahrungen
}

\section{Curriculare und translationsdidaktische Voraussetzungen}

Akademische Formen der Übersetzer- und Dolmetscherausbildung an polnischen Hochschulen werden in unterschiedlichen institutionellen und diversen paradigmatischen Kontexten der didaktisch-methodischen Gestaltung dargeboten. Ihre Repräsentationsformen bilden die translatorischen Studiengänge im Rahmen der Angewandten Linguistik, die translatorischen Spezialisierungen im Bereich der Neuphilologie, die auf der Fachbereichs-, Instituts- und Lehrstuhlebene vertreten sind, und die postgradualen Aufbaustudiengänge. Während das Hauptanliegen der Studiengänge im Rahmen der institutionell etablierten Angewandten Linguistik die Ausbildung von Translatoren ist, stellen die philologisch verankerten translatorischen Spezialisierungen eine Art Bereicherung des philologischen Angebots dar. Nicht zu übersehen ist, dass die Einführung einer translatorischen Spezialisierung in nicht wenigen Fällen für die Institute eine anlockende Funktion in Bezug auf potenzielle Studienkandidaten erfüllen soll.

Im Unterschied $\mathrm{zu}$ autonomen translatorischen Studiengängen werden die Inhalte der Ausbildung sowie der Umfang des didaktischen Angebots vom wissenschaftlichen Profil der Ausbildungsstätte, den institutionellen, organisatorischen und personellen Modalitäten determiniert. Da es im Bereich der Translationsdidaktik keine Bildungsstandards gibt und kein „translatorisches Minimum" vorgegeben ist, also der Anteil der erforderlichen Lehrveranstaltungen bzw. Leistungspunkte aus dem Bereich Translation im Rahmen der Spezialisierungen nicht festgelegt ist, differiert das didaktische Angebot an den 
einzelnen Ausbildungsstätten gravierend (vgl. dazu Ermittlungen von Żmudzki 2008a, 2008b, 2010 und Małgorzewicz 2016). Der Nationale Qualifikationsrahmen für die polnische Hochschulbildung, der 2013 die früheren, die Inhalte der translatorischen Ausbildung eher begrenzenden Bildungsstandards ersetzt hat, sichert die Kompetenzorientiertheit und Offenheit gegenüber neuen und vor allem aktuellen Ausbildungsbedürfnissen des Arbeitsmarktes. Der Vorteil der neuen Regelungen ist in ihrer Flexibilität im Vergleich zur Starrheit der früheren ministeriellen Bildungsstandards sowie in der obligatorischen Kompetenzorientiertheit der Lehrprogramme zu sehen.

Das Postulat der Bologna-Reform, wonach der Programmkonzipierung die Formulierung der Lernziele auf der Ebene des Wissens, der Fähigkeiten und der sozialen Kompetenzen zu Grunde zu legen ist, findet seine unwiderlegbare Begründung. Die Breite des Spektrums möglicher Lernziele lässt sich aus Weinerts Definition der Kompetenz ablesen, sie umfasst:

die bei Individuen verfügbaren oder durch sie erlernbaren kognitiven Fähigkeiten und Fertigkeiten, um bestimmte Probleme zu lösen, sowie die damit verbundenen motivationalen, volitionalen und sozialen Bereitschaften und Fähigkeiten, um die Problemlösungen in variablen Situationen erfolgreich und verantwortungsvoll nutzen zu können (Weinert 2001: 27 f.).

Markant für die dargestellte Kompetenz-Auffassung ist die Fokussierung der Subjektzentriertheit. Im Falle der Translation äußert sich die subjektzentrierte Haltung im Verhältnis des Translators einerseits zu den Sprachen, die er spricht, und andererseits zu seiner Autonomität im Bereich der unternommenen Translationshandlung als Basis, auf der der Translator seine individuelle strategisch-kommunikative Translationskompetenz eigenständig entwickeln und einsetzen kann (Małgorzewicz 2014, 2015). Einen entscheidenden Stellenwert hat in dieser Sphäre das Identitätsbewusstsein des Translators. Die genannten Eigenschaften kommen im authentischen translationsorientierten Handeln zum Tragen, da gerade im Handeln das jeweilige Individuum die der Translationskommunikation adäquaten Handlungsziele vollzieht, und zwar auf eine Art und Weise, welche seinem Wertesystem, seinen Erfahrungen und Haltungen entspricht. Aus dem Gesagten ergibt sich die Forderung an die Translationsdidaktik, realitätsnahe Aufgaben in den Unterricht einzubeziehen. Die von Weinert hervorgehobene Problemlösungskompetenz - also solche Metabereiche der translatorischen Kompetenz wie Reflexion, Selbst- und Fremdkontrolle, das Erwägen mehrerer Lösungsvarianten, das Argumentieren der eigenen Entscheidungen und im Endeffekt die Verantwortungsübernahme für das erarbeitete Translat können in kooperativ angelegten Übersetzungsaufgaben gefördert werden. Das gemeinschaftliche Erarbeiten der Endfassung einer Übersetzung ermöglicht die Konfrontation mit unterschiedlichen Perspektiven und einen interaktiven Wissensaustausch, wodurch auch die Qualität des Translats gesteigert wird. In der 
interaktiven Lernumgebung gründen sich Team- und Kompromissfähigkeit Eigenschaften also, welche im Nationalen Qualifikationsrahmen besondere Beachtung erfahren.

Die Vorteile der kooperativen Übersetzungsmethode wurden von den Autoren des neuen Lehrprogramms für die translatorische Spezialisierung am Institut für Germanistik der Universität Wroclaw erkannt, und das kooperative Arbeitsszenario wurde im neuen Lehrprogramm zu einem der Fächer im letzten Semester des Magisterstudiums konzipiert. Das Fach Kooperatives Übersetzen bildet zusammen mit den Fächern Praktikum im Übersetzungsbüro und Weiterentwicklung der Übersetzerwerkstatt das Modul Praxis des Übersetzers.

$\mathrm{Zu}$ den Prinzipien dieser Lehrveranstaltung wurde die Erfüllung folgender Voraussetzungen erklärt:

- authentische/realitätsnahe Arbeitskontexte (Übersetzungsaufträge),

- authentische Übersetzungstexte,

- Angaben zu Fristen, Textsorte, Auftraggeber, Zweck der Übersetzung, Adressaten der Übersetzung,

- Kleingruppenarbeit (Aufgabenverteilung unter den Gruppenteilnehmern),

- die koordinierende und überwachende Funktion des Dozenten,

- Vorgehen nach einem im Vorfeld festgelegten zeitlichen Ablaufplan der einzelnen Arbeitsschritte,

- individuelles Übersetzen kombiniert mit der Auswertung in der Kleingruppe, Korrekturen, Diskussionen und gemeinschaftlicher Entscheidungsfindung,

- Dokumentation der einzelnen Arbeitsschritte (Protokollführung),

- Zugang zu Hilfsmitteln im Unterrichtsraum, adäquate Medienausstattung (PC, Internetzugang, Beamer, Tafel),

- adäquate, die Spezifik des kooperativen Übersetzens berücksichtigende Methoden zur Leistungsmessung,

- Präsentation der Endfassung der Übersetzung, Evaluierung des Projektes.

Das letzte Semester der translatorischen Ausbildung scheint uns der richtige Moment zu sein, die Form des kooperativen Übersetzens einzusetzen. Das bislang von den Studierenden erworbene Wissen, ihre Fähigkeiten, aber auch Erfahrungen im Übersetzen erlauben es, das Potenzial dieser Methode effektiv auszuschöpfen. Die Metabereiche der translatorischen Kompetenz, die Kritikfähigkeit und das Methodenbewusstsein sind zu diesem Zeitpunkt auch dank der wissenschaftlichen Beschäftigung der Studierenden im Magisterseminar auf einem entsprechend hohen Niveau. Von positiver Bedeutung ist auch die Tatsache, dass die Studierenden, die in dieser Lehrveranstaltung zusammentreffen, einander nicht fremd sind. Sie bilden eine Seminargruppe, die alle übrigen translatorischen Lehrveranstaltungen bereits in derselben Zusammensetzung absolviert hat. Dieser Umstand erleichtert dem Dozenten die Bildung der Kleingruppen. 


\section{Planung und Umsetzung eines kooperativen Übersetzungsprojekts - Untertitelung des Kinderfilms „Lola auf der Erbse“}

Im Juni 2015 wurde am Institut für Germanistik der Universität Wrocław unter der Leitung von Patricia Hartwich das erste Übersetzungsprojekt im Rahmen der Lehrveranstaltung Kooperatives Übersetzen erfolgreich abgeschlossen. SeminarteilnehmerInnen waren 11 Studierende des Masterstudiengangs Germanistik (2. Studienjahr) mit der Spezialisierung Translatorik, die bereits eine Vorbildung über drei Semester in den Bereichen Übersetzen und Dolmetschen absolviert hatten.

Auf der Grundlage des weiter oben vorgestellten Konzepts sollten im Rahmen dieser Lehrveranstaltung vor allem folgende Lehrziele erreicht werden:

- Einführung in das audiovisuelle Übersetzen (a.Ü.) in theoretischer und praktischer Hinsicht - entsprechend dem Grundsatz: Theorie muss praxisrelevant sein;

- Entwicklung von entsprechenden praktischen Fertigkeiten in einer Form des a.Ü. - Voice-over, Untertitelung oder Live-Synchronisation;

- Entwicklung von Kompetenzen, die sich aus der Erfüllung eines authentischen bzw. realitätsnahen Übersetzungsauftrags ergeben (Adressatenorientiertheit, Normgerechtheit des Zieltextes, Einhaltung von Fristen (von der Erarbeitung der einzelnen Teilaufgaben bis hin zur Erstellung des endgültigen Translats);

- Entwicklung von Metakompetenzen: Fähigkeiten zum Reflektieren des eigenen/gemeinsamen Übersetzungsprozesses, damit verbunden: Argumentationsfähigkeit (Begründung der eigenen translatorischen Entscheidungen), kritische Beurteilung eigener sowie fremder Vorschläge/Translate, Korrektur, Fähigkeit zur gemeinsamen Entscheidungsfindung, allgemeine Teamfähigkeit), Problemlösungsstrategien, Recherchestrategien.

In früheren Lehrveranstaltungen wurden von den Dozenten bei den Studierenden häufig ungeeignete Einstellungen und Arbeitsweisen bei der gemeinsamen Bearbeitung von umfangreicheren Aufgaben festgestellt (z.B. bei der gemeinsamen Erarbeitung von Referaten und der gemeinsamen Erstellung von Translaten im Rahmen früherer Übersetzungsprojekte). Die Teamarbeit beschränkte sich in der Regel auf die einfachste Form arbeitsteiligen Vorgehens, nämlich darauf, dass das Referat bzw. der zu übersetzende Gesamttext unter den Gruppenmitgliedern aufgeteilt und die einzelnen Teilergebnisse später zu einem Gesamtergebnis zusammengefügt wurden, sodass im Grunde keine gemeinschaftliche Erarbeitung, Diskussion oder Korrektur erfolgte. Daher bestand eines der Hauptziele der Lehrveranstaltung Kooperatives Übersetzen darin, die Studierenden dazu anzuhalten, dieses Arbeitsmuster 
zu durchbrechen, sodass sie zu der Erkenntnis gelangen, dass das Ganze gewissermaßen mehr sein kann und mehr sein sollte als nur die Summe der einzelnen Teile.

Die Arbeit während des Semesters verlief in folgenden Phasen, auf die im Folgenden näher eingegangen wird:

1. Vorbereitung und Einführung in das a.Ü.,

2. Übersetzung eines aktuellen Kinderspielfilms entsprechend einem konkreten Übersetzungsauftrag,

3. Korrektur (individuell sowie in der Gruppe),

4. Vorführung des Films für eine polnische Schulklasse und Evaluation.

\subsection{Vorbereitung}

Der von der Seminarleiterin vorgeschlagene Kinofilm „Lola auf der Erbse“ in der Regie von Thomas Heinemann erschien erst Mitte des Semesters auf DVD, sodass die Vorbereitungsphase zwangsläufig länger ausfiel, dafür aber bei Erscheinen der DVD abgeschlossen sein musste, damit das Projekt bis Semesterende beendet und einer Schulklasse vorgestellt werden konnte. ${ }^{1}$ Die Vorbereitung verlief wie folgt:

(1) erste Annäherung an die Besonderheiten des a.Ü. durch gemeinsames Übersetzen der Dialogtexte eines deutschsprachigen Kurzfilms - dabei Aktivierung des Vorwissens, Erkennen einiger Spezifika und Probleme beim a.Ü., Diskutieren alternativer Lösungsmöglichkeiten;

(2) Referat einer Studierenden, die sich im Rahmen ihrer Magisterarbeit bereits eingehender mit dem Thema a.Ü. beschäftigt hatte (verschiedene Formen des a.Ü., Filmübersetzung in Polen, geschriebene und ungeschriebene Regeln und Normen bei der Filmübersetzung in Polen, u.a. im Hinblick auf Zeilenanzahl und Länge, max. Zeichenzahl, Einblendungszeit, erforderliche Kürzung der Dialogtexte, Übersetzung von visuellen Textelementen, Übersetzungsstrategien, Umgang mit Metaphern, Vulgarismen usw.);

(3) Annäherung an die technische Realisierung der Untertitelung: Konsultation und Recherche der Studierenden zu kostenfrei nutzbaren Untertitelungsprogrammen - in die nähere Auswahl kamen zwei Programme, die bei der probeweisen Untertitelung eines weiteren deutschsprachigen Kurzfilms während des Unterrichts parallel ausprobiert wurden; vorläufige Entscheidung für die Verwendung des Programms Aegisub; Einarbeitung mehrerer Studierender während der Untertitelungsübung zum Kurzfilm;

(4) Teilnahme der Studierenden am Workshop zum a.Ü., geleitet von Frau Dr. Plewa von der Universität Warschau, die sich sowohl wissenschaftlich-empirisch (vgl. Plewa 2014) als auch praktisch eingehend mit a.Ü. beschäftigt (theoretische

${ }^{1}$ Es sei darauf hingewiesen, dass die einmalige Vorführung des Films keinesfalls kommerziellen oder Werbezwecken diente. 
Einführung und praktische Übungen zu Untertitelung, Voice-over und Synchronisation, Übungen zum Kürzen von authentischen Dialog- und Interviewtexten).

\section{2. Übersetzung}

Nachdem die Studierenden den Film gesehen und sich einstimmig dafür ausgesprochen hatten, ihn im Rahmen des Projekts zu übersetzen, musste das auf DVD vorliegende Format entsprechend den Anforderungen der Software angepasst werden, um ein möglichst effektives Arbeiten zu ermöglichen, d.h. es wurden eine Filmdatei im MP4-Format sowie eine SRT-Datei mit deutschen Untertiteln für Hörgeschädigte extrahiert, sodass die zeitlich bereits platzieren deutschen Untertitel für Hörgeschädigte genutzt und mit den polnischen überschrieben werden konnten, wobei ggf. die Einblendungszeit angepasst, überflüssige Untertitel entfernt und zusätzliche erstellt wurden.

Anschließend wurden die im Film auftretenden sprechenden Personen so innerhalb der Gruppe verteilt, dass jeder Kleingruppe, bestehend aus 2-3 Studierenden, sämtliche Dialogtexte von jeweils 1-4 der insgesamt 15 Figuren zugeteilt wurden. In Vorbereitung auf den Unterricht bereiteten die Studierenden in den entsprechenden Kleingruppen die polnische Übersetzung der sprachlichen ÄuBerungen ihrer Figuren vor. Dieser Vorgehensweise lag zum einen die Annahme zugrunde, dass jede Filmfigur einen eigenen sprachlich-künstlerischen Stil besaß, den es zu erkennen und beim Übersetzen (in unterschiedlichem Maße) zu berücksichtigen galt. Andererseits sollte durch diese Arbeitsweise vermieden werden, dass bereits ganze Dialoge vorgefertigt werden, die anschließend nur noch in der richtigen Reihenfolge zusammengesetzt werden müssen. Somit wurde die Entstehung einer Arbeitsatmosphäre angestrebt, die das gemeinsame Verfassen lebendiger Dialogtexte zu den einzelnen Szenen, das kritische Aushandeln von Lösungen und insgesamt ein gemeinschaftliches problemorientiertes und kreatives Arbeiten ermöglichen sollte.

Während der ersten beiden Treffen erstellten alle Studierenden in chronologischer Abfolge gemeinsam mit der Dozentin die ersten Filmuntertitel. Diese Vorgehensweise erwies sich zwar einerseits als dazu geeignet, dass sich die Studierenden gemeinsam für bestimmte Makrostrategien entscheiden konnten (z.B. die weitgehende Kürzung der Dialogtexte in Anbetracht der jungen, in Bezug auf Untertitel leseunerfahrenen Zielgruppe der 13-jährigen Schüler, der Umgang mit sprachlich inkorrekten Äußerungen des kurdischen Vaters, der Umgang mit visuellen Texten, kulturellen Divergenzen usw.). Zudem konnten in dieser Phase von der Seminarleiterin aufgrund der direkten Kontroll- und Einflussmöglichkeit die Weichen für eine selbstkritische, reflektierende Arbeitsweise in der späteren Kleingruppenarbeit gestellt wurden. Andererseits wurden aber auch 
gravierende Nachteile dieser Arbeitsweise deutlich: die geringe Aktivität einiger Studierender während der Gruppenarbeit sowie der hohe Zeitaufwand bei der Untertitelung (innerhalb von $180 \mathrm{Min}$. Unterricht wurden nur knapp 7 Min. Film untertitelt. Um den Erfolg des Projekts nicht zu gefährden, wurde die Arbeitsweise nun radikal geändert. Jeder Studierende erhielt vorbereitend von der Seminarleiterin eine Übersicht der Szenenfolge mit den jeweils auftretenden sprechenden Personen sowie einen Arbeitsplan für den nächsten Unterricht, dem die zu untertitelnden Szenen mit den darin vorkommenden sprechenden Personen zu entnehmen waren. Entsprechend diesem Plan bearbeiteten die Studierenden während der folgenden Lehrveranstaltungen in zwei Gruppen unterschiedliche Szenen. Die Gruppen arbeiteten zeitgleich in zwei Räumen, um einander nicht zu stören. In jeder Gruppe musste mindestens eine Person anwesend sein, die mit der Bedienung des Untertitelungsprogramms vertraut war, sowie jeweils mindestens ein Vertreter jeder Übersetzerkleingruppe, sodass die Übersetzungen der einzelnen Aussagen während der Gruppenarbeit eingehend erläutert und begründet werden konnten. Die Gruppen führten über ihre Arbeit Protokoll, worin vor allem Unsicherheiten bezüglich der gewählten Übersetzungsvarianten oder Probleme technischer Natur festgehalten wurden. Da Verspätungen oder gar die Abwesenheit einzelner Studierender (wie dies in anderen Lehrveranstaltungen nicht unüblich ist) die Arbeit der gesamten Gruppe behindert hätte, wurde auch das individuelle Verantwortungsbewusstsein der Studierenden gefördert. Wenn sich jemand verspätete, sorgte er bereits vor dem Unterricht dafür, dass der Gruppe zu Unterrichtsbeginn das erforderliche Textmaterial als Arbeitsgrundlage zur Verfügung stand. Die Rolle der Lehrkraft beschränkte sich in dieser Phase vollkommen auf die Vor- und Nachbereitung. Die Gruppenarbeit gestaltete sich aufgrund der geringeren Gruppenstärke äußerst intensiv und effektiv, es entstanden lebhafte Diskussionen. Bereits nach drei Unterrichtseinheiten waren alle Szenen des Films untertitelt.

Während der Übersetzungsphase wurden die Studierenden mit verschiedenartigen Übersetzungsproblemen konfrontiert, z.B. mit der Übersetzung von visuellen Textelementen, mit kulturellen Divergenzen und der Übersetzung von sprachlich inkorrekten Äußerungen des kurdischen Vaters und Sohnes. Zur Veranschaulichung der Übersetzungsprobleme sowie der von den Studierenden gewählten Lösungen werden nachfolgend drei Beispiele vorgestellt.

Beispiel 1: In einer der ersten Szenen des Films hört die strenge, aber gerechte Lehrerin, Frau Kuhbart durch Kopfhörer laute Opernmusik, während sie Klassenaufsätze korrigiert. Von der Musik sichtlich beschwingt, setzt sie unter die mit roten Markierungen übersäte Arbeit von Kevin Barkelt, dem angeberischen Sohn des geldgierigen Barbesitzers, die Note 6. Das Übersetzungsproblem ergab sich hier aus den unterschiedlichen Notensystemen in Deutschland und Polen: Während die Sechs in Deutschland die schlechteste Note ist, ist sie in Polen die 
beste. Dieses Vorwissen konnte bei der Zielgruppe jedoch nicht in jedem Fall vorausgesetzt werden. Um das Verständnis zu sichern und die Komik der Situation zu bewahren, entschlossen sich die Studierenden daher an dieser Stelle einen zusätzlichen Untertitel einzufügen, der die Bedeutung der Note verbal wiedergibt: ocena niedostateczna [Note: ungenügend].

Beispiel 2: In einer anderen Szene zu Beginn des Films „klettert“ der eigentlich seit mehreren Jahren im Leben seiner 12-jährigen Tochter abwesende Vater aus dem auf Lolas Nachttisch stehenden gerahmten Bild und singt für sie ein selbstkomponiertes Schlaflied, woraufhin sie ruhig einschläft. Der deutsche Liedtext zu diesem Einschlafritual wurde von einer Studentin so nachgedichtet, dass sowohl sein origineller Inhalt als auch der Reim als ein typisches Merkmal polnischer Wiegenlieder erhalten geblieben sind (vgl. Tab. 1).

Tab. 1: Nachdichtung des Wiegenliedes (Übersetzung: Zuzanna Mizera, Rückübersetzung P.H.)

\begin{tabular}{|l|l|l|}
\hline \multicolumn{1}{|c|}{ Dt. } & \multicolumn{1}{|c|}{ Pol. } & \multicolumn{1}{c|}{ Rückübersetzung } \\
\hline Jetzt schläfst du ein, & Oczka mruż, & (Mach die) Augen zu, \\
\hline müde wird dein linkes Bein. & Lewa noga chrapie już. & $\begin{array}{l}\text { dein linkes Bein schnarcht } \\
\text { schon. }\end{array}$ \\
\hline Das rechte auch, & I prawa też, & Und das rechte auch, \\
\hline $\begin{array}{l}\text { hungrig knurrt dein dicker } \\
\text { Bauch. }\end{array}$ & twój brzuch chce coś zjeść. & dein Bauch will was essen. \\
\hline Und ist dein Bauch nicht dick, & Jeśli nie jest duży, & Falls er nicht dick ist, \\
\hline $\begin{array}{l}\text { ganz egal, denn morgen gibt } \\
\text { es ein Frühstück }\end{array}$ & $\begin{array}{l}\text { spokojnie, to śniadanie mu } \\
\text { postuży. }\end{array}$ & $\begin{array}{l}\text { bleib ruhig, das Frühstü } \\
\text { wird ihm schon helfen. }\end{array}$ \\
\hline mit Ei und Speck, & Jajeczko zjesz & Du isst ein kleines Ei, \\
\hline $\begin{array}{l}\text { das macht den dünnen Bauch } \\
\text { gleich weg. }\end{array}$ & I brzuch będzie okragły też. & $\begin{array}{l}\text { und auch dein Bauch wird } \\
\text { rund. }\end{array}$ \\
\hline
\end{tabular}

Beispiel 3: Das dritte Beispiel gibt einen kurzen Dialog zwischen dem kurdischen Vater und seinem Sohn wieder, in dem der Sohn die grammatikalisch fehlerhafte deutschsprachige Äußerung seines Vaters verbessert, dieser jedoch auf deren Richtigkeit beharrt und sie logisch begründet (vgl. Tab. 2). Der fehlerhafte Artikelgebrauch im deutschen Originaltext beruht hier auf der Abweichung zwischen dem natürlichen Geschlecht Lolas, das sich im Gebrauch ihres Namens äußert, und dem grammatischen Geschlecht der übergeordneten Kategorie „Mädchen“ ((die) Lola - das Mädchen). Da das Genus des polnischen Wortes „dziewczynka" (dt. Mädchen) feminin ist, musste eine andere Kategorie gefunden werden, um den Sinn der Szene wirkungsäquivalent zu konstituieren. Die Studierenden entschieden sich daher, statt des Femininums „dzwiewczynka“ (dt.: Mädchen) das Neutrum „dziecko“ (dt.: Kind) zu verwenden, wodurch der Sinn der Szene und ihr Witz erhalten geblieben sind. 
Tab. 2: Thematisierung der grammatikalischen Richtigkeit der sprachlichen Äußerung des Vaters im Dialog (Hervorhebungen durch Fettdruck: P.H.)

\begin{tabular}{|l|l|l|l|}
\hline \multicolumn{2}{|c|}{ Deutscher Dialogtext } & \multicolumn{2}{c|}{ Polnische Untertitelung } \\
\hline $\begin{array}{l}\text { Tayip: } \\
\text { (Vater) }\end{array}$ & $\begin{array}{l}\text { Ich muss Ihnen meine Glückwün- } \\
\text { sche sagen für das kluge Lola. }\end{array}$ & Tayip: & $\begin{array}{l}\text { Chciałbym złożyć Pani gratulacje } \\
\text { za „mądre Lolę”. }\end{array}$ \\
\hline $\begin{array}{l}\text { Rebin: } \\
\text { (Sohn) }\end{array}$ & Die. & Rebin: & MĄDRĄ. \\
\hline Tayip: & Wieso die? & Tayip: & Dlaczego „MĄDRĄ”? \\
\hline Rebin: & Die kluge Lola. & Rebin: & MĄDRA LOLA. \\
\hline Tayip: & $\begin{array}{l}\text { Das kluge Lola. Lola ist ein Mäd- } \\
\text { chen. Artikel von Mädchen ist das. } \\
\text { Das Mädchen - das kluge Lola. } \\
\text { Immer muss er alles besser wissen. }\end{array}$ & Tayip: & $\begin{array}{l}\text { MĄDRE Lola. Lola to dziecko. } \\
\text { A jest przecież TO dziecko. TO } \\
\text { dziecko. To MĄDRE Lola. Zaws- } \\
\text { ze musi lepiej wiedzieć. }\end{array}$ \\
\hline
\end{tabular}

\subsection{Korrektur}

Die Seminarleiterin erstellte aus den Ergebnissen der Gruppenarbeit eine vorläufige Gesamtfassung, die jedoch noch mit zahlreichen Fehlern orthografischer, stilistischer und technischer Art behaftet war. Jeder Studierende sollte nun den Film allein „korrektursehen“ und dazu ein individuelles Korrekturprotokoll verfassen, in dem sämtliche Anmerkungen und Verbesserungsvorschläge unter Angabe der entsprechenden Timecodes vermerkt wurden (vgl. Abb. 1). Anschließend folgte eine gemeinsame Korrekturphase (über zwei Lehrveranstaltungen), während der die Ergebnisse der individuellen, sehr gewissenhaft durchgeführten Einzelkorrekturen auf Grundlage sämtlicher Protokolle zusammengetragen, gemeinsam diskutiert und in die Gesamtversion eingearbeitet wurden. In dieser Phase wurden Nutzen und Mehrwert der kooperativen Arbeitsweise für die Gruppenmitglieder besonders deutlich, da von allen zusammen weitaus mehr Fehler entdeckt und diskutiert wurden, als dies einem Einzelnen möglich gewesen wäre. Nach der Übersetzung des Abspanns durch eine Studentin war die Untertitelung des Films abgeschlossen und das Ergebnis konnte vorgestellt und evaluiert werden. 


\section{Korrekturprotokoll}

Name:

\begin{tabular}{|c|c|c|c|}
\hline \multirow[t]{2}{*}{$\begin{array}{l}\text { Szene } \\
\text { Nr. }\end{array}$} & \multirow[t]{2}{*}{$\begin{array}{l}\text { Anfang } \\
\text { der } \\
\text { Szene }\end{array}$} & \multicolumn{2}{|c|}{$\begin{array}{l}\text { Korrekturbedarf } \\
\text { Fehler (semant./stil./orthogr. u. ä.), Ungenauigkeit, Synchronität, fehlender/überflüssiger } \\
\text { Untertitel, zu langer Untertitel usw. }\end{array}$} \\
\hline & & Timecode & Beschreibung \\
\hline 0. & $\begin{array}{l}\text { bis } \\
10: 06\end{array}$ & $\begin{array}{l}0,0: 00: 41.72,0: 00: 43.87 \\
0,0: 02: 32.76,0: 02: 36.79 \\
0,0: 05: 01.16,0: 05: 03.23 \\
0,0: 05: 48.64,0: 05: 50.99 \\
0,0: 05: 52.72,0: 05: 55.07 \\
0,0: 06: 13.64,0: 06: 18.07 \\
0,0: 07: 53.44,0: 07: 58.30\end{array}$ & $\begin{array}{l}\text { Interpunktionsfehler: der Satz sollte mit Auslassungspunkten } \\
\text { beendet werden - jetzt endet der Satz mit einem Punkt } \\
\text { Stilistischer Fehler, mein Vorschlag -> To tak jak z kartą rowerową: } \\
\text { trzeba \N zdać egzamin i dopiero wolno wędkować. } \\
\text { Stil.Fehler, mein Vorschlag ->Zostanę też bez emerytury. } \\
\text { Stilistisch, mein Vorschlag ->To wzbudza publiczne zgorszenie! } \\
\text { Co wzbudza? \N-Zgorszenie! } \\
\text { Interpunktionsfehler-Das Komma ist es zu setzen -> Nie wiem, i tak } \\
\text { to jutro powtórzymy. } \\
\text { Besser mit „Pani Kubart“ -> Dzień dobry Pani Kuhbart! }\end{array}$ \\
\hline 1. & 10:07 & & \\
\hline 2. & $11: 20$ & & \\
\hline 3. & $12: 37$ & & \\
\hline 4. & $13: 26$ & & . \\
\hline 5. & $15: 00$ & $0,0: 14: 12.36,0: 14: 14.19$ & $\begin{array}{l}\text { Orthogr.Fehler „przylądek” muss gross geschrieben werden -> } \\
\text { Gorsza niż kiedyś na Przylądku Horn! } \\
\text { Interpunktionsfehler ->der Satz sollte mit Auslassungspunkten } \\
\text { beendet werden - jetzt endet der Satz mit einem Punkt }\end{array}$ \\
\hline 6. & $15: 54$ & $0,0: 15: 05.48,0: 15: 08.43$ & $\begin{array}{l}\text { Orthogr.Fehler „przylądek” muss gross geschrieben werden }> \\
\text { Myślisz, że Solmsen \Nnaprawdę był na Przylądku Horn? }\end{array}$ \\
\hline 7. & $16: 30$ & & \\
\hline
\end{tabular}

Abb. 1: Auszug aus dem individuellen Korrekturprotokoll einer Studentin 


\subsection{Präsentation des Übersetzungsprodukts und Evaluation}

Zur Filmvorführung im Institut für Germanistik der Universität Wrocław wurde eine Schulklasse eines benachbarten Gymnasiums eingeladen. Sie bestand aus ca. 30 Schülern im Alter von 13 Jahren, die seit einem Jahr Deutsch lernten. Im Anschluss an die Filmvorführung wurde ein Workshop für die Schüler veranstaltet, im Verlauf dessen u.a. überprüft wurde, inwieweit die Schüler den Film mit Hilfe der von den Studierenden erstellten polnischen Untertitel verstanden hatten. Wie sich herausstellte, hat der Film den befragten Schülern, die nach eignen Aussagen bis dahin erst sehr wenige untertitelte Filme gesehen hatten, nicht nur sehr gut gefallen, sondern sie haben ihn auch detailliert verstanden.

Problematisch erwies sich in translationsdidaktischer Hinsicht für die Seminarleiterin die Bewertung der einzelnen Studierenden in Form einer individuellen Note, da die tatsächliche Arbeit der einzelnen Gruppenmitglieder, die sich sicher in unterschiedlichem Maße für das Projekt engagiert hatten, für die Leiterin schwer nach objektiven Kriterien beurteilbar war. Die bei Gruppenprojekten bisweilen eingesetzten Formen der Selbstbeurteilung oder gegenseitigen Beurteilung der Gruppenmitglieder hingegen erschienen aufgrund der in Polen bestehenden Lehr- und Lerntraditionen wenig erfolgversprechend. Bei zukünftigen Projekten sollte daher bereits im Vorfeld ein geeignetes Bewertungssystem nach möglichst objektiven Kriterien erarbeitet und angewandt werden.

Die Bilanz für dieses Übersetzungsprojekt zum kooperativen Übersetzen fiel dennoch insgesamt sehr positiv aus. Im Hinblick auf die anvisierten Lehrziele kann hier der anonyme Kommentar eines oder einer Studierenden angeführt werden, der/die die Arbeit im Rahmen der Lehrveranstaltung in einer anonymen Online-Umfrage unseres Instituts wie folgt bewertet hat: „Te zajęcia okazały się bardzo fajną przygodą - myślę, że dla każdego z nas sporządzenie napisów do filmu było czymś zupełnie nowym. Bardzo dużo można się było nauczyć, przy czym rozwinęliśmy także swoje umiejętności pracowania w grupie, dochodzenia do kompromisów i podejmowania jednoznacznych decyzji.” - „Der Unterricht erwies sich als ein tolles Abenteuer - ich glaube für jeden von uns war das Erstellen von Filmuntertiteln etwas völlig Neues. Man konnte sehr viel dabei lernen, und außerdem entwickelten wir unsere Fähigkeiten, in der Gruppe zu arbeiten, Kompromisse zu finden und klare Entscheidungen zu treffen. ${ }^{\text {2 }}$

2 Die angeführte Beurteilung der Lehrveranstaltung „Kooperatives Übersetzen“ erfolgte anonym durch eine/einen berechtigte/n Studierende/n über das Online-Studienverwaltungssystem der Universität Wrocław USOS (deutsche Übersetzung der Bewertung: P.H.). 


\section{Literatur}

Adamowicz-Grzyb, Grażyna: Kurs przekładu audiowizualnego. Warszawa 2010a.

Adamowicz-Grzyb, Grażyna: Jak redagować napisy do filmów. ABC tlumacza filmowego. Warszawa $2010 \mathrm{~b}$.

Jüngst, Heike: Audiovisuelles Übersetzen. Tübingen 2010.

Kornelius, Joachim / Wencke, Orbán: Kooperatives Übersetzen - ein Beitrag für eine kommunikations-orientierte Übersetzungsdidaktik. In: AREAS-Annual Report on English and American Studies, 34, 2008, S. 489-510.

Małgorzewicz, Anna: Językowe i niejęzykowe kompetencje tlumacza. Próba zdefiniowania celów translodydaktyki akademickiej. In: Lingwistyka Stosowana 11, 2014. S. 1-10.

Małgorzewicz, Anna: Die Autonomie des Translators und ihre Bedeutung für die Translationsdidaktik. In: Weigt, Zenon / Kaczmarek, Dorota / Makowski, Jacek / Michoń, Marcin (Hrsg.): Text-Wesen in Theorie und Analysen. (= Felder der Sprache. Felder der Forschung. Lodzer Germanistikbeiträge). Łódź 2015. S. 75-85.

Małgorzewicz, Anna: Translationsdidaktik in Polen. Aktueller Stand und Perspektiven. Warszawa 2016. [im Druck]

Plewa, Elżbieta: Układy translacji audiowizualnych w ujęciu translatoryki antropocentrycznej. Warszawa 2014. Unveröffentlichte Doktorarbeit.

Tomaszkiewicz, Teresa: Materialy audiowizualne z podpisami a nauczanie języków obcych. In: Lingwistyka Stosowana 5, 2012, S. 109-119.

Tryuk, Małgorzata: Co to jest thumaczenie audiowizualne? In: Przekładaniec 1, 2008, S. 26-39.

Weinert, Franz E.: Vergleichende Leistungsmessung in Schulen - eine umstrittene Selbstverständlichkeit. In: Weinert, Franz, E. (Hrsg.): Leistungsmessung in Schulen. Weinheim 2001. S. 17-32.

Żmudzki, Jerzy: Zum Stand der Translationsdidaktik in den polnischen Germanistikinstituten. (Ein Arbeitsbericht). In: Glottodidactica XXXIV, 2008a, S. 153-171.

Żmudzki, Jerzy: Translationsdidaktik in den polnischen Germanistikinstituten. Realität und Anspruch. In: Grucza, Franciszek (Hrsg.): Translatorik in Forschung und Lehre der Germanistik. Warszawa 2008b. S. 71-96.

Żmudzki, Jerzy: Aktuelle Profile der germanistischen Translationsdidaktik in Polen. In: Małgorzewicz, Anna (Hrsg.): Translation: Theorie - Praxis - Didaktik. (= Studia Translatorica 1). Wrocław 2010. S. 117-136.

\section{Abstracts}

Im Zuge der Umstrukturierung des Masterstudiengangs Germanistik an der Universität Wrocław infolge der 2011 in Polen eingeleiteten Hochschulreform wurde das Studienprogramm für die Spezialisierung „Translatorik“ neu konzipiert und um die projektorientierte Lehrveranstaltung „Kooperatives Übersetzen" erweitert, die im Sommersemester 2015 zum ersten Mal durchgeführt wurde. Ziel dieses Projekts war die auftragsgerechte polnische Untertitelung des deutschen Kinderfilms „Lola auf der Erbse“ (Regie: Thomas Heinemann), wobei die Studierenden sich einerseits mit den spezifischen Anforderungen audiovisueller Translationsformen vertraut machten und andererseits die Möglichkeit erhielten, team- und kommunikationsorientierte Arbeitsformen gewinnbringend für den Lern- und Arbeitsprozess zu nutzen und entsprechende Kompetenzen zu entwickeln.

Schlüsselwörter: Masterstudiengang, Studienprogramm, Translatorik, Kooperatives Übersetzen, audiovisuelle Translation, Film, Untertitelung, Kompetenzen 


\section{Cooperative translation in the process of translating a film - observations and experience in regard to didactic aspects of translation}

In the course of restructuring of the MA programme of German Studies at the University of Wrocław following the university reform introduced in Poland in 2011, the study programme of the translation specialization was redesigned and enriched with the project-oriented seminar called "Cooperative Translation," which was organized for the first time in the summer term of 2015. The objective of this project was to create Polish subtitles suitable for the target audience for the German children's film Lola auf der Erbse (director: Thomas Heinemann). On the one hand, the students familiarized themselves with the technical requirements specific to the audio-visual translation methods. On the other hand, they had an opportunity to develop relevant capabilities and to use team-oriented and communication-oriented types of student work which were profitable for the learning process and the working process.

Keywords: MA programme, study programme, translation studies, cooperative translation, audiovisual translation, film, subtitling, competence

Patricia Hartwich

Uniwersytet Wrocławski

Instytut Filologii Germańskiej

P1. Nankiera $15 \mathrm{~b}$

50-140 Wrocław

Polen

E-Mail: patricia.hartwich@uwr.edu.pl

Anna Małgorzewicz

Uniwersytet Wrocławski

Instytut Filologii Germańskiej

Pl. Nankiera $15 b$

50-140 Wrocław

Polen

E-Mail: anna.malgorzewicz@uwr.edu.pl 